\title{
Electroless Nickel-Phosphorus-Polymer Composite Coatings
}

\author{
O.R.M. Khalifa ${ }^{*}, 1$ and E. Sakr² \\ ${ }^{1}$ Chemistry Department, ${ }^{2}$ Physics Department, Faculty of Girls for Arts, Science \& Education, Ain Shams University. \\ Cairo, Egypt
}

\begin{abstract}
This study examines the use of polyvinyl-pyrrolidone (nitrogen containing compound) (PVP) coating on electroless Ni-P deposit on low steel. Three different average molecular weights of PVP were used, molecular weight 10.000 (PVP-10), molecular weight of, 24.500 (PVP-24.5) and molecular weight 40.000 (PVP-40). Coatings were characterized using X- ray diffraction (XRD). Scanning electron microscope (SEM) was used to determine the surface morphology. The microhardness and corrosion resistance of the composite were studied. It was found that i- the micro hardness decreases in presence of the polymer PVP, ii- the corrosion resistance greatly increases in the presence of PVP and iii- the adhesion of the coatings with the substrate increase in the presence of polymer.
\end{abstract}

Keywords: Electroless, steel, polymer composite, corrosion protection.

\section{INTRODUCTION}

Electroless nickel-phosphorus is widely used in chemical, mechanical and electronic industries because of its corrosion, wear resistance and its inherently uniform coating thickness [1]. Electroless plating allows complex parts to be plated evently and completely even over sharp edges and deep recesses. These would be difficult to plate with electrolytic processes because of current density variation. These systems are autocatalytic (selfcatalyzing) meaning that they will continue to plate into their own deposit after the substrate has been completely covered [24]. In recent years the electroless nickel process has been used in a wide variety of applications in aerospace, automotive, electronics, computers, machinery, nuclear, oil, gas production and value industries. Composite coatings using electroless nickel as the matrix have increased the surface finishing and engineering communications for many years. Composite coatings containing polymers have achieved promising results in improving mechanical properties of coated films. Many articles containing electroless nickel polymer composites have been reported. It was found that Teflon reduces the hardness and wear resistance of the coated film. Shoeibe [5] showed that electroless nickel polymer composite improved the physico mechanical properties of coated film, improves impact resistance, ductility and reduce the hardness of the coated film, also, increases the protection of corrosion. Zhao [6] showed that nickel polymer had anticorrosion properties in $\mathrm{HCl}$ and $\mathrm{NaCl}$. Best corrosion protection of carbon steel and copper results was obtained with polyaniline (Pani) and poly-(orthoxyaniline) (poma) by Verar [7].

\section{EXPERIMENTAL}

\section{II.1. Materials}

Polyvinyl pyrrolidone (PVP) with three different molecular weights of PVP were used (PVP-10) with molecular weight of 10.000 , PVP-24.5 with molecular

*Address correspondence to this author at the Chemistry department Faculty of Girls for Arts, Science \& Education, Ain shams university. Cairo, Egypt; E-mail:dr.omyma@yahoo.com weight of 24.500 and PVP-40 with molecular weight of 40.000. Low carbon mild steel specimens of dimensions 2.4 x $2.4 \times 0.08 \mathrm{~cm}^{3}$ and composition shown in Table 1, were used as substrate.

Table 1. Chemical Composition of Low Carbon Mild Steel

\begin{tabular}{|c|c|c|c|c|c|}
\hline C & Mn & Si & P & Others & Fe \\
\hline \hline 0.260 & 0.600 & 0.080 & 0.008 & $<0.010$ & Rest \\
\hline
\end{tabular}

The low carbon mild steel substrates were mechanically polished, alkali degreased, water rinsed and dried before treatment by the electroless-nickel method in the absence and presence of the various tested PVP.

\section{П.2. Electroless Nickel Process}

\section{I.2.1. Bath Composition}

$\mathrm{NiSO}_{4} ; \quad 7 \mathrm{H} 2 \mathrm{O} \quad(30 \mathrm{~g} / \mathrm{l}), \quad \mathrm{NaH}_{2} \mathrm{PO}_{2} ; \quad \mathrm{H} 2 \mathrm{O} \quad(25 \mathrm{~g} / \mathrm{l})$, $\mathrm{CH}_{3} \mathrm{COONa}$, (15 g/l) Propionic acid-lactic acid (10 g/l), surfactant and PVP (0.2-4 gm/1).

\section{II.2.2. Operating Conditions}

$\mathrm{pH} 4.6$, temperature $80^{\circ} \mathrm{C}$ and the time of coating is $1 \mathrm{hr}$.

\section{I.2.3. Procedure}

The solutions were prepared using reagent-grade chemicals with distilled water and the bath was at constant temperature $80^{\circ} \mathrm{C}$ during the electroless plating process. The substrate was immersed in absence and presence of PVP.

\section{I.2.4. Mechanical Tests}

The Ni-P coated steel substrate was subjected to mechanical testing methods including: Vickers microhardness under $50 \mathrm{gm}$ load. An average of five replicate samples was recorded.

\section{II.2.5. Morphology}

SEM unit was used to demonstrate the surface morphology. The examination was carried out on different 
samples employing an accelerating voltage of $30 \mathrm{kV}$. Samples were mounted using carbon paste to ground them.

\section{II.2.6. Corrosion Test}

Corrosion behavior was estimated from potentiodynamic polarization measurements on the electroless films. The electrolyte used was artificial seawater $(\mathrm{NaCl} 3.5 \%)$ solution.

The electrochemical properties of the composite coating were investigated using corrosion measurements system model 350 A (Console) with a scan rate $0.5 \mathrm{mV} / \mathrm{sec}$. A saturated calomel electrode (SCE) was used as reference electrode.

\section{U. RESULTS AND DISCUSSION}

\section{U.1. Coating Composition}

Analysis of the coated film is as follows $\mathrm{P}=9.52 \%$ and $\mathrm{Ni}=90.48 \%$ as determined by $\mathrm{X}$-ray micro-analysis.

\section{U.2. Mechanical Properties}

\section{III.2.1. Microhardness Measurements}

The hardness of electroless nickel phosphorus and nickel polymer composite coating has been studied and the data are given in Table $\mathbf{2}$ showing that the hardness decreases in presence of PVP.

Table 2. Hardness Values for Electroless Nickel Phosphorus and Nickel Polymer Composite Coating

\begin{tabular}{|c|c|}
\hline Type of Coating & Hardness Before Heat Treatment (VH50) \\
\hline \hline Ni-P & 454 \\
Ni-P-PVP 40.0 & 330 \\
Ni-P-PVP 24.5 & 285 \\
Ni-P-PVP 10.0 & 249 \\
\hline
\end{tabular}

\section{UI.2.2. Adhesion}

All specimens were cut carefully across the section using a thin diamond cutter. The edge cut was observed, first visually then microscopically on the mounted and polished sections. The results indicate that the adhesion is increased in the presence of polymer (PVP). This is because, the polymers used have high molecular weight and interact with the nickel and improve its adhesion properties with the substrates.

\section{II.2.3. Film Thickness}

The dry film thickness of the electroless nickel phosphorus polymer composite coating ranged from 9 to $12 \mu \mathrm{m}$.

$\mathrm{X}$ - ray diffraction patterns of the as plated electroless nickel polymer composites are shown in Fig. (1) and the corresponding peak position with grain size are given in Table 3. All the diffraction patterns had only a single broad peak at 44.5-2 $\theta$. The corresponding peak position and crystalline size are given in Table 3 .

From Fig. (1) it is evident that the peak broadness has been decreased compared to peak obtained for Ni-P i.e. Nipolymer composites had sharper peaks. Grain size is also increased. Also, it indicates that the crystal microstructures of the composite coatings are mainly amorphous.

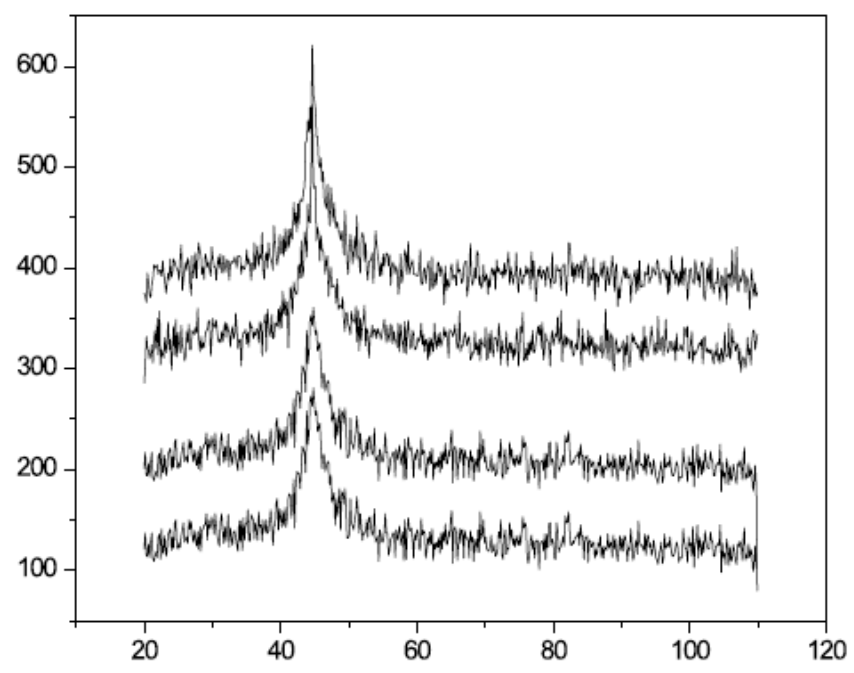

Fig. (1). X-ray diffraction pattern of as plated electroless coatings (a) Ni-P (b) PVP-10 (c) PVP-24.5 (d) PVP-40.

Table 3. Peak Position (20) and Crystalline Size (from DebyeScherrer Formula) for Electroless Nickel Phosphorus Composite Coatings

\begin{tabular}{|c|c|c|}
\hline Type of Coating & Peak Position & Grain Size $(\mathrm{nm})$ \\
\hline \hline Ni-P & 44.5 & 3.6 \\
\hline Ni-PVP 40 & 44.5 & 4.85 \\
\hline Ni-PVP 24.5 & 44.5 & 4.57 \\
\hline Ni-PVP-10 & 44.5 & 4.32 \\
\hline
\end{tabular}

Surface morphology of nickel polymer composite shows that nickel polymer composite, to be nodular type and coarse grained Ni- PVP 10 is the most compact nodules to be cluster, as shown in Fig. (2).

\section{UI.2.4. Corrosion and Polarization Measurements}

Electrochemical potentiodynamic polarization experiments were applied in $3.5 \% \mathrm{NaCl}$ solution to measure the corrosion resistances of low carbon mild steel alloy and as-deposited Ni-PVP10, Ni-PVP24.5 and Ni-PVP40 coatings. For comparison, the corrosion properties of low carbon mild steel alloy coated by $\mathrm{Ni}-\mathrm{P}$, deposited from standared bath and containing the same content of $\mathrm{Ni}$ and $\mathrm{P}$ have been studied Fig. (3). The electrochemical parameters obtained from the polarization curves for uncoated low carbon mild steel and the coated alloy by Ni-PVP10, NiPVP24.5, Ni-PVP40 and Ni-P (blank) are shown in Table 4.

It is evident from the results of Table 4 that Ni-PVP10 coating layer provides effective protection $(\% \mathrm{P})$ for low carbon mild steel alloy. This may be due to the higher mobility of shorter polymeric chain compared to those of larger molecular weight, since PVP-40 is four times higher in comparison to that of the lowest one PVP 10. Also, the protection $(\% \mathrm{P})$ of the coated layer increases with decreasing the molecular weight of the polymer i.e. the protection $(\% \mathrm{P})$ increases in the order:

Ni-PVP10 > Ni-PVP24.5 > Ni-PVP40 > Ni-P. 

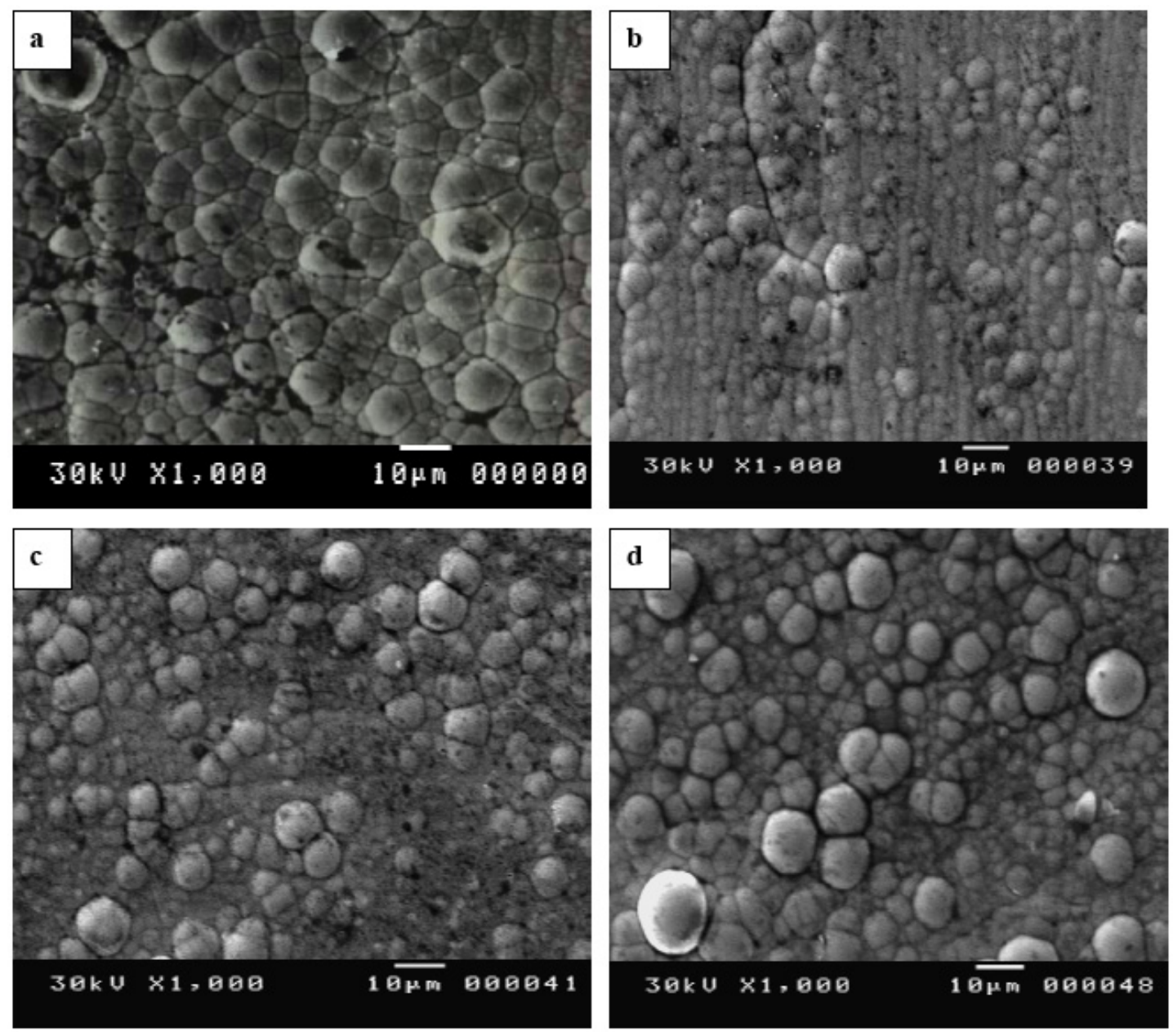

Fig. (2). Scanning electron microscope of electroless nickel-phosphorus composite coating with polymer (a) Ni-P (b) Ni- PVP-10 (c) NiPVP-24.5 (d) Ni- PVP-40.

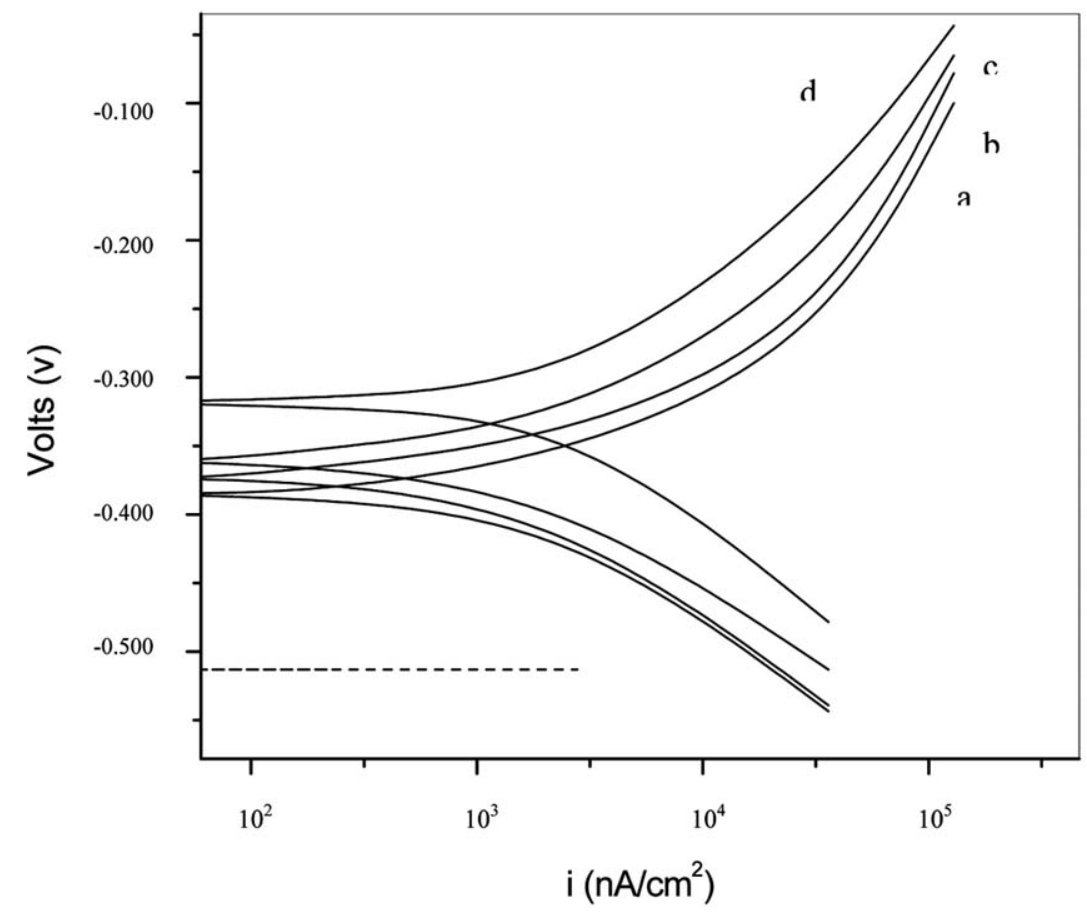

Fig. (3). Potentiodynamic polarization curves of as plated electroless coatings in 3.5\% sodium chloride solution (a) Ni-P (b) PVP, 40.000 (c) PVP, 24.500 (d) PVP, 10.000 coatings in $3.5 \%$ sodium chloride solution. 
Table 4. Electrochemical Data from Tafel Curve Carried Out in 3.5\% NaCl of Different Composite

\begin{tabular}{|c|c|c|c|r|r|}
\hline Type of Coating & MPY & $E_{\text {corr }}(\mathbf{V})$ & $\mathbf{I}_{\text {coor }} \mathbf{n A} / \mathrm{cm}^{2}$ & \%Protection Efficiency $(\% \mathbf{P})$ & $\mathbf{R}_{\mathrm{p}}$ \\
\hline \hline Blank & 6.2 & -0.510 & $4.77 \times 10^{4}$ & 59.8 \\
Ni-P & 3.09 & -0.382 & $2.38 \times 10^{4}$ & 50 & $1.27 \times 10^{3}$ \\
Ni+PVP40 (4 g/l) & 0.715 & -0.372 & $5.67 \times 10^{3}$ & 58 & $50.36 \times 10^{3}$ \\
Ni+PVP24.5 (4 g/l) & 0.613 & -0.364 & $5.6 \times 10^{3}$ & $5.5 \times 10^{3}$ \\
Ni+PVP10 (4 g/l) & 0.537 & -0.318 & $4.0 \times 10^{3}$ & 91.33 \\
\hline
\end{tabular}

From Table 4 all nickel polymer composites had good corrosion resistance in sodium chloride; this is due to the fact that nitrogen containing compounds have good corrosion protective properties due to the lone pair of electrons on the nitrogen atom (present in polyvinyl pyrrolidone). However because Ni-PVP 10 is the most protective composite and so we study the variation of the corrosion protection with the variation of concentration of PVP-10 in $3.5 \% \mathrm{NaCl}$ solution.

Fig. (4) shows potentiodynamic polarization curves with different concentrations of PVP-10 and the corresponding data are shown in Table $\mathbf{5}$.

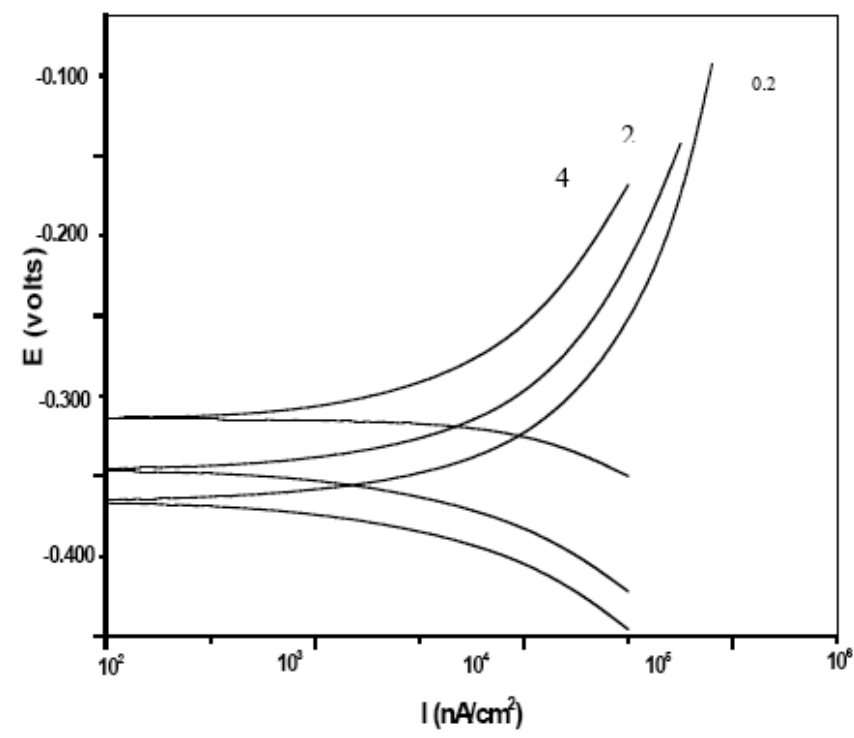

Fig. (4). Potentiodynamic polarization curves of PVP at different concentration $(4,2.0,0.2) \mathrm{g} / 1$ in $0.2 \mathrm{M} \mathrm{NaCl}$.

It is evident from the results of Table $\mathbf{5}$ and Fig. (5) that the protecting efficiency $(\% \mathrm{P})$ of $\mathrm{Ni}-\mathrm{PVP}-10$ is decreased with the decrease of the concentration of PVP-10.

From the results of Tables $\mathbf{4}$ and $\mathbf{5}$, it is evident that the protection efficiency $(\% \mathrm{P})$ of the composites increased with decreasing the molecular weight of the polymer PVP- 10> PVP-24.5> PVP-40. This is due to the higher mobility of shorter polymeric chain compared to those of larger molecular weight, since PVP-40 is four times higher in comparison to that of the lowest one PVP 10. As mentioned above the polymers interact with nickel and improve the adhesion of nickel with the surface of the substrate. The improvement of the film characteristics (i.e good surface coverage with the polymer complex film) enhances the corrosion protection properties.

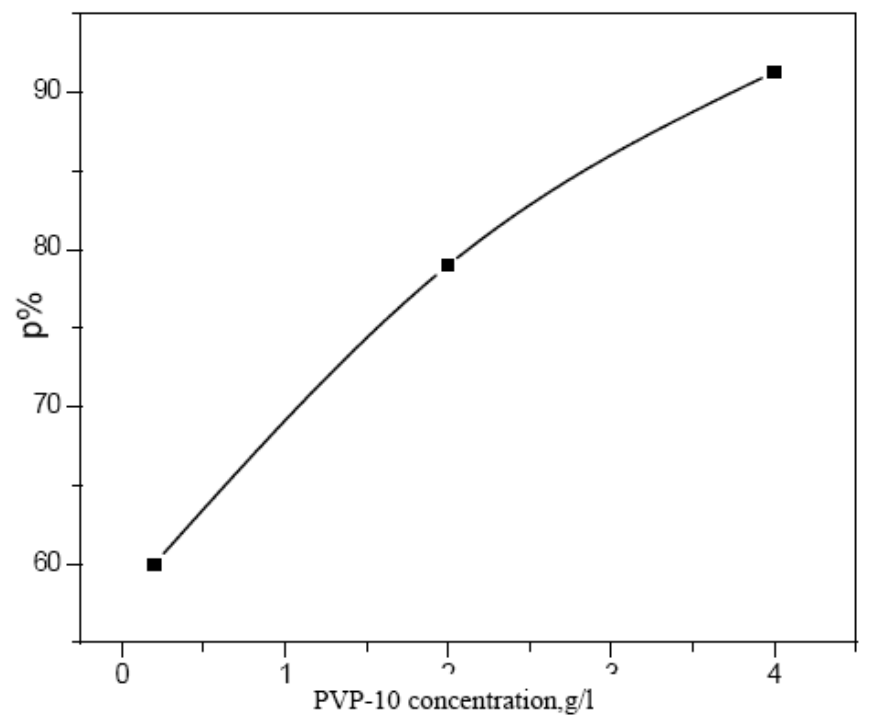

Fig. (5). Relation between the percentage protection (\% P) and concentration. of PVP-10.

\section{CONCLUSION}

XRD results revealed that the entire as plated composite had a single broad Ni (iii) peak. Scanning electron microscope showed that as plated composites exhibit smooth nodular structure. The results indicate that the adhesion increased in presence of the polymer. Also, the as plated composites showed excellent corrosion resistance due to the good surface coverage with the polymer complex film. The protection efficiency of the composites increased with decreasing the molecular weight of the polymer as: PVP-10 > PVP-24.5 > PVP-40 > Ni-P.

Table 5. Electrochemical Data from Tafel Curves Carried Out in 3.5\% NaCl in Presence of Different Concentrations of Ni-PVP10

\begin{tabular}{|c|c|c|c|c|c|}
\hline Concentrations of PVP & Mpy & Ecorr & Icorr & \% P & R \\
\hline \hline Blank & 6.200 & -0.510 & $4.77 \times 10^{4}$ & & 59.8 \\
Ni-P-PVP10- 4g/l & 0.537 & -0.318 & $4.1 \times 10^{4}$ & 91.3 \\
Ni-P-PVP10- 2g/l & 1.300 & -0.327 & $1.00 \times 10^{4}$ & 79.0 & $6.66 \times 10^{3}$ \\
Ni-P-PVP10- 0.2g/l & 2.470 & -0.336 & $1.90 \times 10^{4}$ & 60.0 & $1.35 \times 10^{3}$ \\
\hline
\end{tabular}




\section{REFERENCES}

[1] Straffelini G, Colombo D, Molinari A. Surface durability of electroless Ni-P composite deposits. Wear 1999; 236: 179-88.

[2] Goldstein AW, Rostoker W, Schossberger F, Gutzeil G. Structure of chemically deposited nickel. J Electrochem Soc 1957; 104(2): 104-10.

[3] Graham AH, Lindsay RW, Read HJ. The structure and mechanical properties of electroless nickel. J Electrochem Soc 1965; 112(4): 401.

[4] Shoeibe MA. Nickel electroless plating on aluminum and its alloys. M.Sc Thesis, Faculty of Science, Cairo University, 1978.
Shoeibe MA, Mokhtar SM. Mechanical and corrosion protection properties of electroless nickel-polymer composite coatings. Met Finishing 1998; 96: 58-9.

[6] Zhao Q, LiuY. Electroless Ni-Cu-P-PTFE composite coatings and their anticorrosion properties. Surf Coating Technol 2005; 200: 2510-4.

[7] Vera R, Schirebler R, Cury P, Del Rio R, Romero H. Corrosion protection of carbon steel and copper by polyaniline and poly(ortho-methoxyaniline) films in sodium chloride medium. Electrochemical and morphological study. J Appl Electrochem 2007; 37: 519.

Received: December 18, 2008

(C) Khalifa and Sakr; Licensee Bentham Open.

This is an open access article licensed under the terms of the Creative Commons Attribution Non-Commercial License (http://creativecommons.org/licenses/by$\mathrm{nc} / 3.0 /$ ) which permits unrestricted, non-commercial use, distribution and reproduction in any medium, provided the work is properly cited. 